\title{
The prognostic role of IDH mutations in homogeneously treated patients with anaplastic astrocytomas and glioblastomas
}

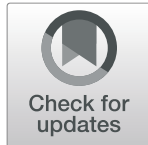

\author{
Arne Christians ${ }^{1}$, Antonia Adel-Horowski ${ }^{2,3}$, Rouzbeh Banan ${ }^{1}$, Ulrich Lehmann ${ }^{4}$, Stephan Bartels ${ }^{4}$, Felix Behling ${ }^{5}$,
} Alonso Barrantes-Freer ${ }^{6,7}$, Christine Stadelmann ${ }^{6}$, Veit Rohde ${ }^{2}$, Florian Stockhammer ${ }^{2,8}$ and Christian Hartmann ${ }^{1 *}$

\begin{abstract}
The detection of IDH mutations in patients with diffusely infiltrating malignant astrocytomas resulted in substantial modifications in the concept of WHO classification of these tumors. An important underlying observation was that patients with anaplastic astrocytomas (AA) without IDH mutation had a clinical course similar to that of patients with glioblastomas (GBM). The underlying observations of the German Glioma Network and NOA-04, however, were based on mixed patient cohorts. While most GBM patients received combined radiochemotherapy, patients with AA usually had radiotherapy or chemotherapy only. This intrinsic shortcoming of the study raised the question of whether patients with AA, IDH wildtype, WHO grade III, might have better prognosis if treated with combined radiochemotherapy than patients with GBM receiving the same combination therapy. Thus, the question remains whether the established histopathological grading criteria for malignant astrocytomas in the absence of an IDH mutation are still important if neither vascular proliferation nor necrosis are detectable. All patients in the cohort investigated here with the diagnosis of AA or GBM were subjected to a combined radiochemotherapy according to the Stupp protocol independently of the histopathological diagnosis. Thus, the analysis of these patients allows to clarify whether patients with AA, IDH wildtype, WHO grade III have a prognosis similar to that of GBM, IDH wildtype, WHO grade IV, even under equivalent therapeutic conditions. We determined the IDHI and IDH2 status by sequencing, the MGMT status by pyrosequencing after bisulfite treatment and the EGFR status of the patients by FISH. In fact, the patients with the histopathological diagnosis of an AA IDH wild-type under similar aggressive therapy showed a comparable and therefore no better prognosis (median overall survival (mOS) 16 months) than patients with a GBM (mOS 13 months). Instead, patients with an AA and an IDH mutation receiving the same therapy had a mOS of 54 months. Thus, it can be concluded that in the absence of an IDH mutation, the established histopathological grading criteria 'necrosis' and 'vascular proliferation' actually lose their prognostic significance. If, on the other hand, patients with malignant astrocytomas and an IDH mutation are examined, there is still a difference between patients with necrosis and/or vascular proliferation and those whose tumors do not show such characteristics. Accordingly, in patients with malignant astrocytomas with IDH mutation it can be concluded that a histological differentiation between AA IDH mutated and GBM IDH mutated remains beneficial from a prognostic perspective.
\end{abstract}

Keywords: IDH1, IDH2, MGMT, EGFR, Prognosis, Grading, Anaplastic astrocytomas, Glioblastomas

\footnotetext{
* Correspondence: hartmann.christian@mh-hannover.de; https://www.mhhannover.de/pathologie-neuropat.html

'Department of Neuropathology, Institute of Pathology, Hannover Medical

School (MHH), Carl-Neuberg-Str. 1, D-30625 Hannover, Germany

Full list of author information is available at the end of the article
}

(c) The Author(s). 2019 Open Access This article is distributed under the terms of the Creative Commons Attribution 4.0 International License (http://creativecommons.org/licenses/by/4.0/), which permits unrestricted use, distribution, and reproduction in any medium, provided you give appropriate credit to the original author(s) and the source, provide a link to the Creative Commons license, and indicate if changes were made. The Creative Commons Public Domain Dedication waiver (http://creativecommons.org/publicdomain/zero/1.0/) applies to the data made available in this article, unless otherwise stated. 


\section{Introduction}

Diffusely infiltrating malignant astrocytomas are differentiated into anaplastic astrocytomas WHO grade III (AA) and glioblastomas WHO grade IV (GBM) due to the negative prognostic significance of the histopathological biomarkers 'necrosis' [1] and 'microvascular proliferation' [2] in all editions of the WHO classification of brain tumors [3-7]. According to the revised fourth edition of the WHO classification of brain tumors from 2016, malignant astrocytomas are furthermore differentiated based on their IDH status into the group of anaplastic astrocytomas WHO grade III with/ without IDH mutation (AA IDH-mut/AA IDH-wt) and into the group of glioblastomas WHO grade IV with/ without IDH mutation (GBM IDH-mut/GBM IDH-wt) [6]. This differentiation of tumors according to IDH status was based on the observation that a positive IDH status correlated with a better prognosis for the patients both within the group of AA [8] and within the group of GBM [9]. Furthermore, it could be demonstrated that patients with AA IDH-wt had a worse prognosis than patients with GBM IDH-mut [10]. Genetically and epigenetically it was also observed that AA and GBM with/without IDH mutation are two different entities. It emerged that most histopathologically defined AA IDH-wt had the epigenetic and genetic signature of GBM IDH-wt. Morphologically diagnosed GBM IDH-mut, on the other hand, were genetically characterized as tumors from the group of IDH mutated diffuse astrocytomas [11-14]. For example, GBM IDH-wt carry EGFR amplifications in around 45\% [15] and TERT mutations in up to $80 \%$ [16] while astrocytomas with an IDH mutation exhibit such alterations in a rather low number [12].

Patients with GBM show a significantly better response to chemotherapy with alkylating substances such as temozolomide when the MGMT promoter is methylated [17]. The detection of an IDH mutation correlates with MGMT methylation: almost all patients with an IDH mutation also show MGMT promoter methylation [18]. The link between IDH mutations and MGMT promoter methylation is presumably due to the competitive inhibition of the alpha-ketogluterate-dependent dioxygenase TET2 by the oncometabolite-2'-hydroxgluterate. As a result, a global hypermethylation of the genome of tumor cells with an IDH mutation occurs [19] and the gCIMP genotype becomes detectable [20]. In this context, it should be noted that the MGMT promoter is also subject to this global hypermethylation [18]. In the absence of an IDH mutation, however, MGMT promoter methylation is found in about $40 \%$ of all GBM patients. In patients with AA IDH-wt, methylation of the MGMT promoter is observed with approximately the same frequency. Accordingly, the MGMT status predicts the presumed success of chemotherapy with temozolomide only in those patients with AA IDH-wt [18].

According to the EANO guideline for the treatment of patients with diffuse gliomas [21], combined temozolomide and radiotherapy followed by temozolomide maintenance treatment is recommended upon diagnosis of GBM regardless of IDH status at age under 70 years, the so-called 'Stupp protocol' [22]. Patients with a AA IDH-mut are advised to receive primary radiotherapy followed by temozolomide or PCV maintenance treatment. In patients diagnosed with AA IDH-wt, however, radiotherapy alone is recommended for those patients with a negative $M G M T$ methylation status and the GBM protocol is recommended for patients with a positive MGMT methylation status.

One of the main observations that led to the concept of different therapeutic recommendations is the missing significant prognostic difference between patients with GBM and AA when both entities are defined by the absence of IDH mutation [10]. However, due to the retrospective concept of this trial, GBM patients treated with the more aggressive Stupp regimen were compared with AA patients who received mostly chemotherapy or radiotherapy alone. The question remains whether patients with IDH wild-type malignant astrocytomas without histological evidence of necrosis and/or vascular proliferation, i.e. with AA IDH-wt, do not differ prognostically under the same therapy from patients diagnosed with GBM IDH-wt. This raises the question whether the established histopathological criteria for grading of AA and GBM in the absence of IDH mutations still have a relevant prognostic significance. Furthermore, it could be assumed that patients with AA IDH-wt live longer as a result of a more aggressive treatment according to the Stupp protocol than patients who received predominantly a monotherapy, as the patients of the cohort studied so far [10]. In such a scenario, it would have to be concluded that the histopathological grading criteria for the differentiation of malignant astrocytic tumors are still valid independent of the IDH status.

Patients diagnosed with AA and GBM at the University Medical Center in Göttingen received the same adjuvant treatment recommendations for several years. Thus, this patient cohort is ideal for a multivariate analysis of prognostic factors especially regarding the question if histopathological detection of prominent microvascular proliferation and/or necrosis still have a prognostic significance in times of molecular IDH diagnostics.

\section{Material and methods \\ Patient collective}

In this retrospective analysis, all patients with the diagnosis of a AA or GBM were identified who i.) underwent surgery at the Department of Neurosurgery at the University Medical Center Göttingen between 1997 and 
2011, ii.) presented with an initial lesion, iii.) received adjuvant radiation therapy and chemotherapy with temozolomide, iv.) had clinical follow-up data and v.) had sufficient formalin-fixed paraffin embedded (FFPE) tumor tissue available for further investigations. Age, preoperative Karnofsky Performance Scale (KPS), extent of resection (EOR), overall survival (OS) were retrieved from the medical records. OS was the primary endpoint of the analysis. FFPE tumor tissue of each tumor was collected from the biobank of the Institute of Neuropathology of the University Medical Center Göttingen. Three experienced neuropathologists $(\mathrm{CS}, \mathrm{CH}, \mathrm{RB})$ reevaluated the samples according to the recent definition criteria of the WHO classification [6]. The study was approved by the Ethics Committee of the University Medical Center Göttingen. Cases with incomplete medical datasets or insufficient amounts of viable tumor tissue for further tissue analysis were excluded from the study.

\section{DNA extraction}

FFPE tissue samples were cut into 11 serial sections each. One section per set was subsequently H\&E stained. The stained tissue sections were evaluated by an experienced neuropathologist (RB) and areas consisting of at least $80 \%$ tumor cells were marked. The corresponding areas on the unstained sections were then scraped into sample tubes, deparaffinized and subjected to DNA extraction with the DNeasy Blood \& Tissue Kit (Qiagen, Hilden, Germany). DNA concentration of the resulting solution was quantified using a FLUOstar Omega plate reader photometer (BMG Labtech $\mathrm{GmbH}$, Ortenberg, Germany).

\section{IDH1 and IDH2 mutation analysis}

The $I D H 1$ and $I D H 2$ mutation status was determined by Sanger sequencing. PCR amplification of the target region was performed by mixing $50 \mathrm{ng}$ of extracted tumor DNA as template, $10 \mu \mathrm{l}$ of HotStar Taq 2X Mastermix (Qiagen), $1 \mu \mathrm{l}$ of the respective forward and reverse primers $(10 \mathrm{pmol}$ each) and high purity water to a final volume of $20 \mu \mathrm{l}$ (PCR primers - IDH1 forward: CGGTCTTCAGAGAAGCCATT, IDH1 reverse: GCAAAATCACATTATTGCCAAC, IDH2 forward: AGCCCATCATCTGCAAAAAC, IDH2 reverse: CTAGGCGAGGAGCTCCAGT). The PCR cycling protocol consisted of an initial activation step at $95^{\circ} \mathrm{C}$ for $5 \mathrm{~min}$, followed by 30 cycles of denaturation at $95^{\circ} \mathrm{C}$ for $30 \mathrm{~s}$, annealing at $60^{\circ} \mathrm{C}$ for $30 \mathrm{~s}$ and elongation at $72^{\circ} \mathrm{C}$ for $60 \mathrm{~s}$, concluding with a final elongation step at $72^{\circ} \mathrm{C}$ for $10 \mathrm{~min}$. The PCR products were checked by agarose gel electrophoresis. Five microliters of PCR product were mixed with one microliter of Illustra ExoProStar One-Step enzyme mix (GE Healthcare, Buckinghamshire, UK) and incubated at $37^{\circ} \mathrm{C}$ for $20 \mathrm{~min}$, followed by inactivation at $80^{\circ} \mathrm{C}$ for 15 min. The samples were then mixed with $6 \mu \mathrm{l}$ of $I D H 1 / 2$ forward primers, respectively, and sent to an external sequencing service provider (GATC, Konstanz, Germany).

\section{MGMT analysis}

The MGMT promoter methylation status was determined by pyrosequencing [23]. The tumor DNA was subjected to bisulfite conversion using the EZ DNA Methylation-Gold Kit (Zymo Research, Irvine, CA, USA). The bisulfite converted DNA was then used as PCR template. For PCR amplification, $100 \mathrm{ng}$ of bisulfite converted DNA was added with $0.1 \mu$ l Platinium Taq Polymerase (Thermo Fisher Scientific, Waltham, MA, USA), $1 \mu$ of each PCR primer (10 pmol), $1 \mu \mathrm{l} 10 \mathrm{mM}$ dNTP (Thermo Fisher Scientific), 2,5 $\mu \mathrm{l}$ PCR buffer, $2,5 \mu \mathrm{l} \mathrm{MgCl} 2(25 \mathrm{mM})$ and high purity water to a final volume of $25 \mu$ l. The PCR primers MGMT_1i5: GTTTYGGATATGTTGGGATAG, MGMT_3i3’: AACC ACTCRAAACTACCACC, MGMT_3i3'bio: Biotin-AAC CACTCRAAACTACCACC and MGMT_1i5'bio: BiotinGTTTYGGATATGTTGGGATAG were used. Because MGMT PCR primers span CpG sites that can be modified through the bisulfite conversion the primer solution consists of an equimolar mix of oligonucleotides with $\mathrm{C} / \mathrm{T}(\mathrm{Y})$ or G/A (R) nucleotides at the position of the CpG sites. The PCR cycling program was composed of an initial activation step at $95^{\circ} \mathrm{C}$ for $5 \mathrm{~min}$, followed by 45 cycles of denaturation at $95^{\circ} \mathrm{C}$ for $30 \mathrm{~s}$, annealing at $60^{\circ} \mathrm{C}$ for $45 \mathrm{~s}$ and elongation at $72{ }^{\circ} \mathrm{C}$ for $30 \mathrm{~s}$, concluding with a final elongation step at $72^{\circ} \mathrm{C}$ for $5 \mathrm{~min}$. The PCR products were checked by agarose gel electrophoresis. Pyrosequencing was performed on a PyroMark Q96 MD system (Qiagen) with PyroMark Gold Q96 reagents (Qiagen) using the primers MGMT-pyro1: TGGTGAGTGTTTGGGT and MGMT-pyro1R: CCAAACACTCACCAAAT. For the sample preparation process, $5-10 \mu \mathrm{l}$ of PCR product were mixed with streptavidin-coated sepharose beads, followed by strand separation and washing with the vacuum prep tool (Qiagen). The bead-bound ssDNA was then mixed with $5 \mu \mathrm{l}$ of sequencing primer $(0.5 \mu \mathrm{M})$ per well and annealed by heating to $80^{\circ} \mathrm{C}$ for $2 \mathrm{~min}$ and subsequent cooling to room temperature. The sequencing results were analysed with PyroMark MD software (Qiagen).

\section{EGFR analysis}

The EGFR status was determined using the Zytovision SPEC EGFR/CEN7 Dual Color Probe FISH assay (Zytovision, Bremerhaven, Germany). The green fluorochrome labeled EGFR probe hybridizes to the $7 \mathrm{p} 11.2$ region encompassing the EGFR region, the orange labeled CEP7 probe hybridizes to the alpha satellite centromere region of chromosome 7 (7p11.1-q11.1). Slides with probe mix were co-denatured at $60^{\circ} \mathrm{C}$ for $1 \mathrm{~h}$ and next hybridized at $37^{\circ} \mathrm{C}$ for $16-24 \mathrm{~h}$ on a ThermoBrite (Abbott Molecular, Abbott Park, IL, USA). Sample pretreatment and post- 
hybridization washes were performed according to the recommendation of the FISH probe manufacturer (Zytovision). Slides were evaluated using a fluorescence microscopy with orange, green and DAPI compatible filters. FISH signal counts for orange and green were counted for a total of at least 100 nuclei in the solid tumor area and an EGFR/CEP7 ratio was calculated. EGFR amplification was defined by an $E G F R / C E P 7$ ratio $\geq 2.0$. Polysomy with an $E G F R / C E P 7$ ratio $<2.0$ or an EGFR/CEP7 ratio $\geq 2.0$ in less than $15 \%$ of the tumor cell nuclei was defined as no EGFR amplification [24].

\section{Statistical analysis}

Overall survival (OS) was defined as the primary endpoint and was calculated from the day of first surgery to the date of death. OS was estimated by the Kaplan Meier method using the Logrank test. A $p$-value less than 0.05 was considered to be of statistical significance. Cox regression analysis was applied to determine the independent prognostic impact of the IDH mutation status, MGMT methylation status as well as pure histopathological diagnosis (AAI, GBM), age at diagnosis (dichotomized at 65 years), preoperative clinical status (KPS, dichotomized at 70\%) and extend of resection (GTR, STR). The software OrignPro v9.5.1 (Northampton, MA) and JMP 14.2.0 (Cary, NC) were used for statistical analysis.

\section{Results}

\section{Clinical characteristics}

A total of 139 patients were identified fulfilling the inclusion criteria. The median age was 63 years (AA: 50 years, GBM: 65 years), the male to female ratio was 1.4 to 1 (AA: 1.3, GBM: 1.5). In summary, 103 patients received a gross total resection (GTR), 30 patients a subtotal resection (STR), 5 patients had only a biopsy taken and in one case no information about the extent of surgery was available. The median overall survival was 14 months (AA: 41 months, GBM: 13 months) and the median KPS prior to surgical resection was 70 (AA: 80, GBM: 70).

\section{Neuropathological and molecular characteristics}

After histopathological assessment of all 139 cases, 116 tumors were graded as GBM (83.5\%) and 23 as AA (16.5\%). Sequencing revealed that 19 cases were IDH1 mutated, mostly $\mathrm{R} 132 \mathrm{H}$ mutations (18 cases) and one R132C mutation. No IDH2 mutations were detected while one case of GBM could not be sequenced for IDH mutations. Thirteen IDH mutations were found in AA (56.5\%), $6 \mathrm{IDH}$ mutations in GBM (5.2\%). This resulted in a distribution of 13 AA IDH-mut, 10 AA IDH-wt, 6 GBM IDH-mut and 110 GBM IDH-wt.
In only 2 out of 139 cases the MGMT status could not be determined, both of them GBMs. In 63/137 cases (46\%) the MGMT promoter was methylated while 74/ 137 cases (54\%) an unmethylated MGMT promoter was found. A $M G M T$ promoter methylation was found in 20 of the 23 analysable AA (87\%), in 3/23 cases no MGMT methylation was seen (13\%). In two GBM MGMT analysis was not possible, in $43 / 114$ cases $M G M T$ methylation was found (38\%), while in $71 / 114$ cases the MGMT promoter was not methylated (62\%). In 18/19 of the AA and GBM with IDH mutation a methylated MGMT promoter was found (95\%), in one case (an AA) no MGMT promoter methylation was detectable despite an IDH mutation (5\%). EGFR analysis was successfully performed in $136 / 139$ cases. In $78 / 136$ cases (57\%) no amplification, in 58/136 tumors (43\%) an EGFR amplification was observed. In 53/114 GBM with a successfully determined EGFR status an amplification was observed (46\%). An high level of EGFR amplification was identified in 5/10 (50\%) of patients with an AA IDH-wt, thereby allowing the designation of a 'Diffuse astrocytic glioma, IDH-wildtype, with molecular features of glioblastoma, WHO grade IV' according to cIMPACTNOW update 3 [25]. None of the tumors with an EGFR amplification showed an IDH mutation. Within the group of 58 tumors with an EGFR amplification MGMT promoter methylation was evident in 24 cases (41\%). In $37 / 76$ cases without EGFR amplification MGMT promoter methylation was observed (49\%) Table 1.

\section{Survival analysis}

In the univariate analysis of all patients with a malignant astrocytoma, age below 65 years $(p<0.001$; Fig. 1a; Table 2) and a KPS above $70(p<0.001$; Fig. 1b) were associated with a longer OS. However, no link between OS and sex (Fig. 1c) or GTR and STR was observed. Patients with the histopathological diagnosis of an AA performed significantly better regarding overall survival compared to patients diagnosed with a GBM $(p<0.0001$; Fig. 1d).

All patients with an AA or GBM demonstrated a significant longer $\mathrm{OS}$ in case of $M G M T$ promoter methylation ( $p<0.001$; Fig. 2b; Table 2). Analysing OS in context of the MGMT status depending on histology, patients with $M G M T$ promoter methylation and an AA showed the longest OS (median OS 41,5 months), while patients with an unmethylated MGMT status and a GBM had the shortest OS (median OS 12 months). Patients with MGMT promoter methylation and a glioblastoma showed an OS in between (median OS 15 months). Because only in three cases the combination of an unmethylated MGMT promoter with the diagnosis of an AA was found, no conclusions regarding this groups could be undertaken (median OS 18 months). Comparing 
a

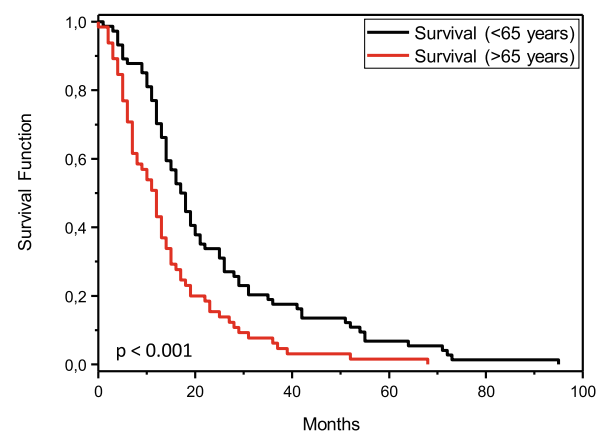

c

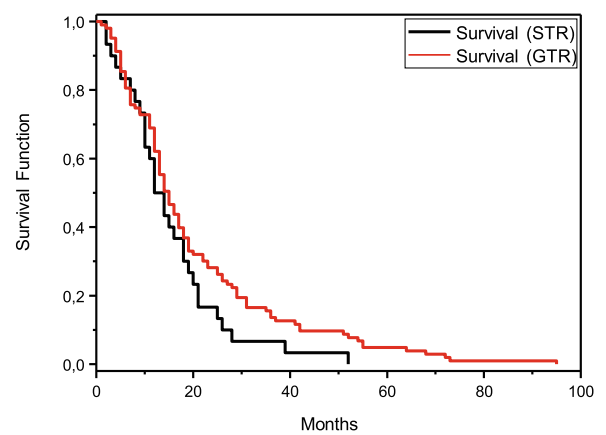

b

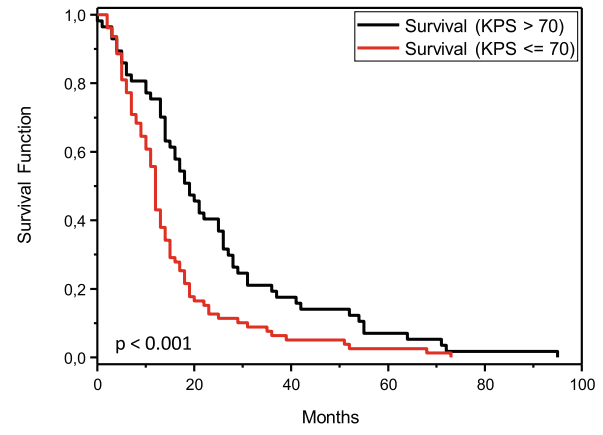

d

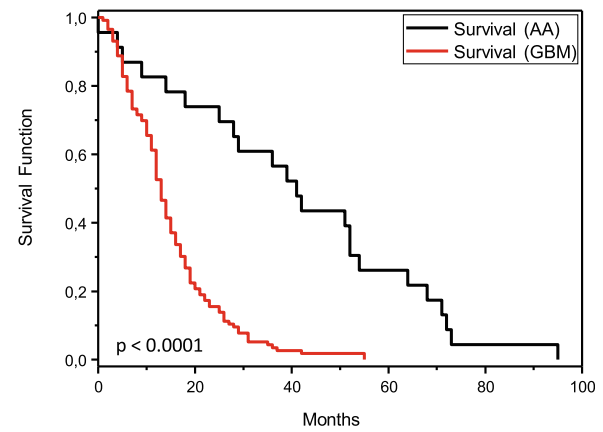

Fig. 1 Kaplan Meier plots a series of 139 patients with malignant astrocytomas, which shows an association between (a) overall survival and an age below and above 65 years. b A significance is also found in the cohort between a Karnofsky Performance Scale (KPS) below and above 70. c The univariate analysis of the extent of tumor resection (EOR) did not show a significant difference, yet in multivariate analysis EOR appeared as an independent prognostic factor. $\mathbf{d}$ However, there is a clear association between the pure histopathological diagnosis of anaplastic astrocytoma (AA) and a glioblastoma (GBM)

only patients with a GBM in respect to MGMT status only a trend towards a better OS for those with promoter methylation was found $(p=0.08)$. Furthermore, no significant difference regarding the OS was seen between patients with an AA and a positive or negative MGMT status $(p=0.4)$. A clear difference in OS was identified between patients with an AA and a positive MGMT status and patients with a GBM and a positive MGMT status $(p<0.00001)$. In a pooled analysis of all four groups a clear prognostic significance of a methylated MGMT status was found ( $p<0.000001$; Fig. 2e).

All patients with a AA or GBM demonstrated significantly longer $\mathrm{OS}$ in case of an IDH mutation $(p<0.0001$; Fig. 2a; Table 2). Furthermore, in the group of patients with AA IDH mutations were associated with a longer OS $(p<0.0001)$. However, the small number of 6 patients with an IDH-mutated GBMs allowed a statistical evaluation with only very limited validity. Next, we did not find a significant difference between patients with an AA IDH-wt and all GBM patients and subjects with a GBM IDH-wt. Patients with an AA IDH-mut had a prolonged OS compared to patients with a GBM IDHmut $(p<0.05)$. In summary, the Kaplan Meier figure of all four groups more or less showed very similar plots for patients with GBM IDH-wt, AA IDH-wt and GBM IDH-mut compared to patients with AA IDH-mut. The differences in OS across the whole group of patients were highly significant $(p<0.0001$; Fig. 2 c). A similar effect was observed in a combined analysis of four subgroups as well when only patients younger than 65 years were analysed ( $p<0.00001$; Fig. 2f). Pooling all malignant astrocytic tumors with available IDH and $M G M T$ status three molecular groups allowed further analysis regarding OS: IDH-mutated and MGMT methylated, IDH-wildtype and MGMT methylated and IDHwildtype and MGMT not methylated. Because only one tumor showed the molecular signature IDH-mutated and MGMT not methylated this variant was not further evaluated. A clear prognostic difference between the molecular subgroups IDH-mutated and MGMT methylated versus IDH-wildtype and MGMT methylated was observed $(p<0,00001)$. No significance was detected between the groups IDH-wildtype and MGMT methylated versus IDH-wildtype and $M G M T$ not methylated but a trend for a longer OS in the first molecular group was seen $(p=0.06)$. Combining the analysis of all 3 molecular 
Table 1 Patients characteristics

\begin{tabular}{|c|c|c|}
\hline & $\begin{array}{l}\text { AA } \\
n=23\end{array}$ & $\begin{array}{l}\text { GBM } \\
n=116\end{array}$ \\
\hline \multicolumn{3}{|l|}{ Age in years } \\
\hline Median & 50 & 65 \\
\hline Range & $25-74$ & $26-81$ \\
\hline Age $(>65,<=65)$ & 6,17 & 63,53 \\
\hline Gender (male/female) & $13,10(1.30: 1.00)$ & $69,47(1.49: 1.00)$ \\
\hline \multicolumn{3}{|l|}{ Extent of resection } \\
\hline GTR & $14(61 \%)$ & $89(77 \%)$ \\
\hline STR & $5(22 \%)$ & $25(22 \%)$ \\
\hline Biopsy only & $4(17 \%)$ & $1(1 \%)$ \\
\hline N.d. & 0 & $1(1 \%)$ \\
\hline $\operatorname{KPS}(>70,<=70$, n.d. $)$ & 15 (65\%), $6(26 \%), 2$ (9\%) & $42(36 \%), 73(63 \%), 0$ \\
\hline $\mathrm{IDH}+, \mathrm{IDH}-$ & $13(57 \%), 10(43 \%)$ & $6(5 \%), 110(95 \%)$ \\
\hline MGMT+, MGMT-, MGMT n.d. & 20 (87\%), 3 (13\%), 0 & $43(37 \%), 71(61 \%), 2$ (2\%) \\
\hline $\mathrm{IDH}+\&$ MGMT+ & $12(52 \%)$ & $6(26 \%)$ \\
\hline IDH- \& MGMT- & $2(7 \%)$ & $71(61 \%)$ \\
\hline $\mathrm{IDH}+\& \mathrm{MGMT-}$ & $1(4 \%)$ & 0 \\
\hline IDH- \& MGMT+ & $8(35 \%)$ & $37(32 \%)$ \\
\hline EGFR+, EGFR -, EGFR n.d. & 5 (50\%), 5 (50\%), 0 & $53(46 \%), 61(54 \%), 3(2 \%)$ \\
\hline
\end{tabular}

AA anaplastic astrocytoma, GBM glioblastoma, GTR gross total resection, STR subtotal resection, n.d. not determined, KPS Karnofsky Performance Scale, IDH+ IDH1 or IDH2 mutation, IDH- no IDH1 or IDH2 mutation, MGMT+ promoter methylation, MGMT- no promoter methylation, EGFR+ amplification, EGFR- no amplification

subgroups a clear association was found as well $(p<0$, 0000001, Fig. 2d). No link between EGFR amplification and OS was found by analysing all tumors, all GBM and all AA IDH-wt, respectively (Table 2). However, comparing AA with and without EGFR amplification a prognostic effect was evident $(p<0.05)$.

The multivariate analysis showed an independent prognostic significance of the variables gross total resection (GTR) $(\mathrm{p}<0.05)$, anaplastic astrocytoma $(p<0.0001)$ and IDH mutation $(p<0.0001)$ when all patients were evaluated. The presence of a methylated MGMT promoter remained insignificant as well in the multivariate analysis. Age at diagnosis below 65 and a KPS status of 70 or more were identified as prognostic factors in the univariate analysis. However, the multivariate analysis did not indicate that they are independent prognostic factors. If patients who only received a biopsy $(n=5)$ were excluded, the variables gross total resection $(p<0.05)$, anaplastic astrocytoma $(p<0.0001)$ and IDH mutation $(\mathrm{p}<0.05)$ remained as independent prognostic factors.

\section{Discussion}

The observation that the IDH status in patients with malignant astrocytic tumors has a significant prognostic relevance [8-10, 26-28] has led in recent years to significant modifications in the classification [6] and treatment of these tumors [21]. For instance, the authors of the current
EANO guideline [21] recommend radiotherapy for patients with $\mathrm{AA}$ and an IDH mutation, followed by temozolomide or PCV-based chemotherapy. Patients with AA IDH-wt, on the other hand, are recommended to receive radiotherapy alone if their MGMT status is negative, combined radiochemotherapy followed by temozolomide treatment if the MGMT methylation status is positive. The IDH status, on the other hand, has no consequences with regard to therapy recommendations for patients with GBM who are to be treated at an age below 70 years according to the Stupp protocol [22]. Interestingly, however, there are clear studies that provide a different therapy recommendation for patients with AA with and without IDH mutation [8], but not for the recommendation to treat patients with AA IDH-wt (and MGMT promoter methylation) identically to GBM patients. However, the underlying observation that these are patients of the same genetic entity with a similar prognosis was based on a pooled data set of NOA-04 and GGN [10]. At that time, the patients under investigation were treated by a regimen based solely on the histological diagnosis, which differed between WHO grade II and WHO grade IV gliomas. The aim of this study is now to assess whether the overall survival of patients with AA IDH-wt is comparable to that of GBM patients with identical therapy according to the Stupp protocol.

The clinical and molecular characteristics of the patients analysed in this cohort are within the range of 
Table 2 Survival characteristics of patients

\begin{tabular}{|c|c|c|c|c|c|}
\hline & & $\begin{array}{l}\text { mOS } \\
\text { in months }\end{array}$ & & $\begin{array}{l}\text { mOS } \\
\text { in months }\end{array}$ & $p$ \\
\hline Age & $<65$ & 17.5 & $>65$ & 12 & $<0.001$ \\
\hline KPS & $>70$ & 19 & $<70$ & 12 & $<0.001$ \\
\hline Sex & M & 15 & $\mathrm{~F}$ & 13 & n.s. \\
\hline EOR & GTR & 15 & STR & 13 & n.s. \\
\hline Histo & AA & 41 & GBM & 13 & $<0.0001$ \\
\hline MGMT & MGMT+ & 20 & MGMT- & 12 & $<0.001$ \\
\hline MGMT AA & MGMT+ & 41,5 & MGMT- & 18 & n.d. \\
\hline MGMT GBM & MGMT+ & 15 & MGMT- & 12 & n.s. \\
\hline $\mathrm{IDH}$ & $\mathrm{IDH}+$ & 51 & $\mathrm{IDH}-$ & 13 & $<0.0001$ \\
\hline IDH AA & $\mathrm{IDH}+$ & 54 & $\mathrm{IDH}-$ & 16 & $<0.0001$ \\
\hline IDH GBM & $\mathrm{IDH}+$ & 11 & $\mathrm{IDH}-$ & 13 & n.s. \\
\hline EGFR & EGFR+ & 14 & EGFR- & 14 & n.s. \\
\hline EGFR AA & EGFR+ & 9 & EGFR- & 42 & $<0.05$ \\
\hline EGFR GBM & EGFR+ & 14 & EGFR- & 12 & n.s. \\
\hline IDH/MGMT & $\mathrm{IDH}+/ \mathrm{MGMT+}$ & 46,5 & IDH+/MGMT- & 64 & n.d. \\
\hline IDH/MGMT & $\mathrm{IDH}+/ \mathrm{MGMT+}$ & 46,5 & IDH-/MGMT+ & 15 & $<0,00001$ \\
\hline IDH/MGMT & $\mathrm{IDH}+/ \mathrm{MGMT+}$ & 46,5 & IDH-/MGMT- & 12 & n.s. \\
\hline IDH/MGMT & IDH-/MGMT+ & 15 & IDH+/MGMT- & 64 & n.d. \\
\hline IDH/MGMT & IDH-/MGMT+ & 15 & IDH-/MGMT- & 12 & n.s. \\
\hline IDH/MGMT & IDH+/MGMT- & 64 & IDH-/MGMT- & 12 & n.d. \\
\hline
\end{tabular}

mOS median overall survival in months, $p$ p-value by Logrank test with a value less than 0.05 indicating statistical significance, Histo histological diagnosis, $A A$ anaplastic astrocytoma, GBM glioblastoma, GTR gross total resection, STR subtotal resection, $M$ male, $F$ female, n.s. not significant, n.d. not determined, KPS Karnofsky Performance Scale, EOR extent of resection, $I D H+I D H 1$ or IDH2 mutation, IDH- no IDH1 or IDH2 mutation, $M G M T+$ promoter methylation, $M G M T$ - no promoter methylation, EGFR+ amplification, EGFR- no amplification

established data on these tumor entities. The CBTRUS report for the years 2011-2015 states a median age of 65 years for GBM patients and 50 years for patients with AA [29]. The median average age of the patients examined here was 65 years and 53 years, respectively. The ratio of male to female was 1.36:1.00 for GBM (presented cohort 1.49:1.00) and 1.20:1.00 (1.30:1.00) [29]. In the present study, $57 \%$ of patients with AA and $5 \%$ of patients with GBM had IDH mutations, while 60 and $7.2 \%$, respectively, were found in a comparable study [10]. In $87 \%$ of patients with AA (reference: $55 \%$ ) and in 37\% (reference: $47 \%$ ) of GBM patients $M G M T$ promoter methylation was found [10]. While we found an EGFR amplification in $46 \%$ of the patients with a GBM, a frequency of $35-45 \%$ is reported in the literature [6]. We identified EGFR amplification in $50 \%$ of patients with AA. Other studies reported a lower rate of less than $10 \%$ [30]. However, it must be taken into account that with the low number of cases of 23 patients with AA investigated here, a sampling error seems conceivable as explanation. Established risk factors regarding overall survival for patients with malignant diffusely infiltrating astrocytomas are the histopathological criteria of necrosis and vascular proliferation, the age of the patient, the extent of resection, the KPS as well as molecular characteristics like MGMT and IDH status [21]. In the univariate analysis, we were able to confirm all these characteristics as risk factors in the cohort examined here except for the extent of resection (see Figs. 1 and 2). The extent of resection was subjectively assessed by the surgeon immediately following the operation. However, neuroradiological postoperative quantification was not performed. Accordingly, it seems conceivable that the subjective assessment by various neurosurgeons did not lead to consistent data, so that the assessment of the extent of resection was erratic and could therefore not be identified as a risk factor. In summary, however, it can be concluded that the series of patients examined is representative for the question addressed.

Several interesting observations result from the molecular data of the cohort of patients with malignant astrocytic tumors investigated here. As one of the most important observations it could be documented that patients with AA IDH-wt showed a similar overall survival as patients with GBM IDH-wt despite combined radiochemotherapy. With regard to this aspect it could be expected that a more aggressive therapy according to the Stupp protocol would result in a prolonged overall 
a

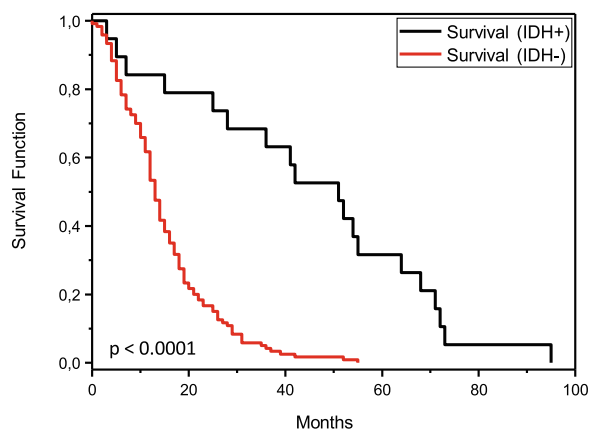

C

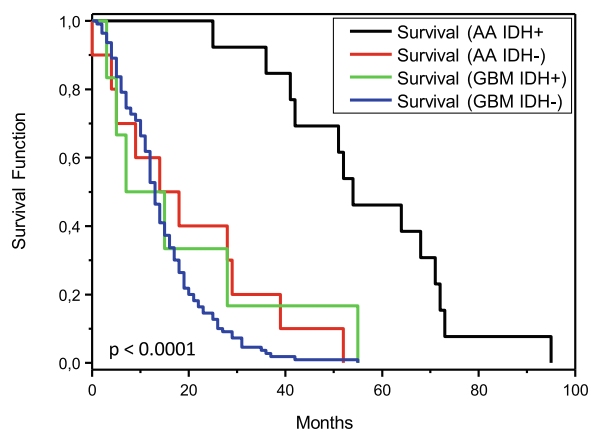

e

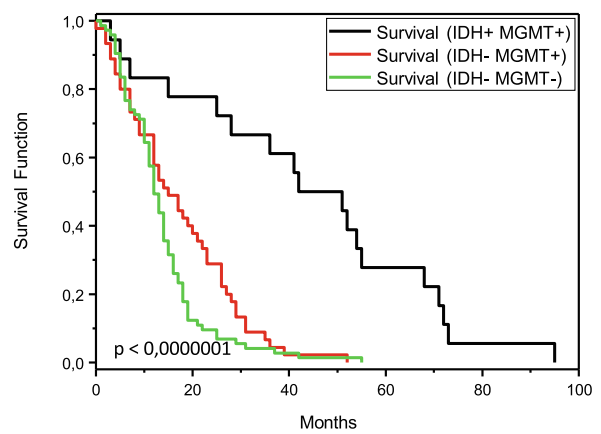

b

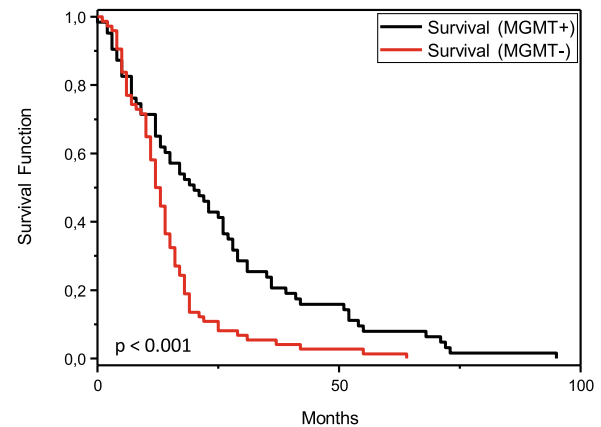

d

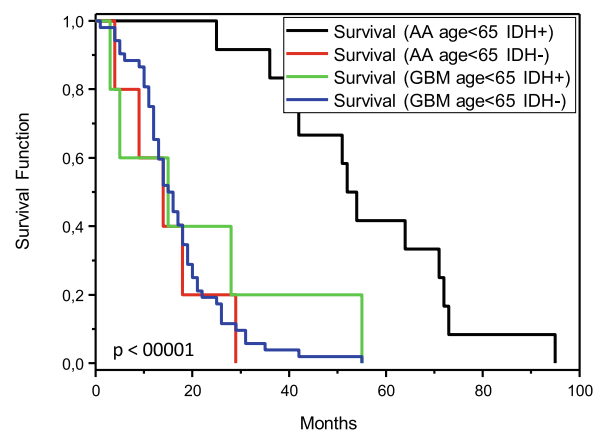

f

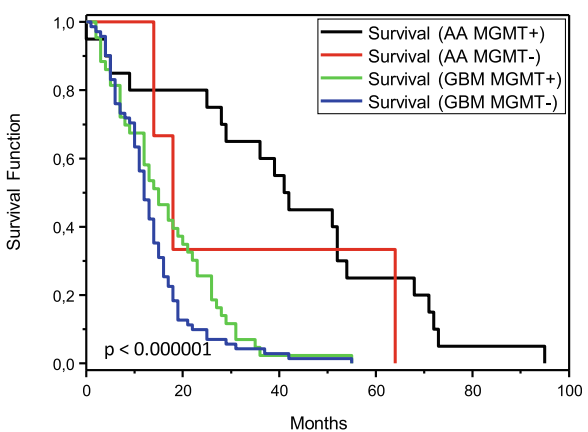

Fig. 2 Kaplan Meier plots derived from 139 patients diagnosed with an anaplastic astrocytoma or glioblastoma. a There is an association between overall survival and IDH status and (b) between overall survival and MGMT status. c There is a significant effect in the combination of histopathological diagnosis and IDH status. Patients with anaplastic astrocytoma and IDH mutation show better overall survival than patients with anaplastic astrocytoma without IDH mutation and patients diagnosed with glioblastoma with/without IDH mutation. The overall survival of the patients of the latter three subgroups appears similar in the Kaplan Meier analysis. $\mathbf{d}$ The same significant effect regarding histopathological diagnosis and IDH status became evident when only patients were subject to analysis below the age of 65 years. e Combining IDH and MGMT status patients with a positivity for both markers performed better than those patients with a negative IDH status independent from the MGMT status. Because only one tumor showed the combination IDH+ and MGMT- this group was not plotted. f Analyzing the histopathological diagnosis in combination with the MGMT status patients with an anaplastic astrocytoma with MGMT promoter methylation showed a better overall survival than the other groups. However, only 3 patients with an anaplastic astrocytoma revealed a negative MGMT promoter status so no further conclusion could be drawn from this group. AA: anaplastic astrocytoma, GBM: glioblastoma, IDH+: IDH1 or IDH2 mutation, IDH-: no IDH1 or IDH2 mutation, MGMT+: promoter methylation, MGMT-: no promoter methylation

survival for patients with AA IDH-wt. In the comparative study [10], only $20 \%$ of these patients received combined radiochemotherapy, while in the cohort analyzed here all patients diagnosed with AA IDH-wt were treated accordingly. Despite this more aggressive therapy, no increase in overall survival was documented. On the other hand, the survival plot line of patients with AA IDH-wt was even closer to that of patients with GBM in the Kaplan Meier graph (Fig. 2c). Various conclusions can be drawn from this observation. Firstly, it can be concluded that AA 
IDH-wt actually genetically correspond to GBM IDH-wt, since patients with these tumors show a very similar overall survival under comparable therapeutic conditions. Secondly, the question also arises whether the additional cytotoxic side effects of a more aggressive therapy justify the lack of increase in overall survival for patients with AA IDH-wt. However, it must be critically noted that this conclusion is based on a rather small number of patients in the present study. Prospective studies with a larger number of patients are necessary to generate sufficient data regarding this issue.

Furthermore, it is remarkable that in terms of overall survival, patients with GBM IDH-mut do not differ from patients diagnosed with GBM IDH-wt or AA IDH-wt. In the corresponding comparative study [10], the Kaplan Meier plot showed a plot line of these patients above the line of patients with AA IDH-wt. However, no significance was apparent in the comparative study either. On this basis, it was discussed to potentially treat patients with a GBM IDH-mut less aggressively than patients with a GBM IDH-wt. The data presented here argue rather against such a suggestion to modify the established therapy according to the EANO guideline [21]. However, it must be emphasized that a small number of patients with this genetic alteration is the basis of the conclusion.

With regard to the question whether the established histopathological criteria for grading of malignant astrocytic tumors in the IDH age remain important under identical therapy, it can be concluded from the available data that this is still the case to some extent. There is a significant difference in OS between patients with AA IDH-mut and GBM IDH-mut. Therefore, it can be concluded for this group of malignant astrocytomas that the detection of necrosis and vascular proliferation remains important for the evaluation of the prognosis. A different picture emerges when we consider patients with AA and GBM that show no IDH mutation. The corresponding Kaplan Meier plots for OS of these patients are very similar and no significant difference can be shown. Accordingly, it can be concluded from the data that for patients with malignant diffusely infiltrating astrocytomas without IDH mutation, the histopathological grading criteria necrosis and vascular proliferation are only of minor importance. It should be noted that recently homozygous $C D K N 2 A$ deletions were identified as prognostic markers for malignant astrocytic tumors with IDH mutation [31]. This molecular marker was not evaluated in the present study. However, an analysis of the $C D K N 2 A$ status would be of interest for a subsequent study in order to evaluate its significance in the context of classical histopathological grading criteria.

The frequently published prognostic data on patients with AA IDH-wt indicate that these tumors are genetically GBM IDH-wt $[8,10,26-28]$. While this hypothesis is probably true for the largest number of such tumors, it cannot be excluded that a fraction of histopathologically defined AA corresponds indeed to another entity. For example, an unrepresentative biopsy of an anaplastic ganglioglioma, an anaplastic pleomorphic xanthoastrocytoma or a GBM with an H3F3A G34R/V mutation may also appear as a diffuse infiltrating AA IDH-wt. If in this combination only the absence of an IDH mutation in tumors with a malignant astrocytic morphology that do not exhibit necrosis and/or vascular proliferation were determined as definition criteria, the above-mentioned prognostically more favorable tumors would incorrectly be assigned to a WHO grade IV. For this reason, cIMPACTNOW Update 3 requires the detection of an EGFR amplification, a TERT promoter mutation or a complete gain of chromosome 7 combined with a complete deletion of chromosome 10, in addition to the two criteria 'diffuse astrocytoma' and 'no IDH mutation', in order to be able to establish the diagnosis 'Diffuse astrocytic glioma, IDHwildtype, with molecular features of glioblastoma, WHO grade $I V$ [25]. For this reason, we determined the EGFR status of all patients. Indeed, an EGFR amplification was found in 5/10 anaplastic AA IDH-wt, so that these tumors can subsequently be assigned to a WHO grade IV according to cIMPACT-NOW update 3. Due to the remaining very small number of $5 \mathrm{AA}$ IDH-wt without EGFR amplification, we refrained from further statistical evaluation.

While methylation of the MGMT promoter had a clear prognostic significance in the entire cohort of malignant astrocytic tumors, isolated analysis of patients diagnosed with GBM revealed only a trend in this direction. Most studies document a prognostic significance of MGMT promoter methylation for GBM in the context of combined radiochemotherapy $[9,17]$. Other publications, however, did not report a prognostic significance of MGMT promoter methylation [32-34]. Various reasons can be debated for the discrepant observation. Tthere are no generally accepted and standardized assays for $M G M T$ analysis, so that especially in the context of different analyzed $\mathrm{CpG}$ sites, distinct results can emerge. The MGMT assay we use covers 14 CpG sites [23], which also cover the region, which are mostly given as reference [17]. Accordingly, we assume that the assay we use sufficiently determines the MGMT status. Furthermore, it is discussed that a different material quality (frozen tissue versus FFPE tissue) can lead to different MGMT results. In summary, however, the most likely explanation for our observations is a slight sampling error which leads to the lack of significance while still a trend can be observed.

The present study has various weaknesses. The number of 139 patients diagnosed with malignant astrocytoma included in the study appears to be rather limited, considering that only 13 patients diagnosed with an AA 
IDH-mut, 10 patients diagnosed with an AA IDH-wt and 6 patients diagnosed with a GBM IDH-mut are covered. Furthermore, it is a retrospective analysis. Accordingly, a prospective study would be recommended, which should include a significantly larger number of patients with the above-mentioned diagnoses. The CATNON study is expected provide a large dataset when IDH data become available [35]. However, no GBM patients are covered in the CATNON study, so that no conclusions can be drawn from these study data regarding the histopathological grading criteria for malignant astrocytic tumors. Another deficit of the study presented here are incomplete clinical data regarding progressionfree survival (PFS), which did not allow sufficient analyses. Especially in the context of identical therapeutic conditions, interesting data could have been generated by the correlation of PFS and histopathological and molecular markers. In this respect, the CATNON study may also provide further information when IDH data will be available [35]. Furthermore, we always used preoperative imaging information to determine contrastenhancing regions to ensure that the most malignant tumor regions were resected in case of subtotal resection or biopsy. However, we do not have adequate postoperative imaging in all cases so we cannot exclude the possibility that a few AA might be undergraded GBM. In addition, even in case of totally resected tumors we cannot rule out the possibility that we missed necrotic areas or microvascular proliferates in some tumors diagnosed as $\mathrm{AA}$, due to non-representative sampling of the tissue for histological processing.

In summary, the study presented here shows that patients diagnosed with an AA IDH-wt have basically the same prognosis as patients diagnosed with a GBM IDHwt when treated according to the Stupp protocol. Therefore, the concept of the current WHO classification to stratify patients with malignant astrocytomas according to the IDH status can be confirmed [6]. The data generated here can also be quoted to confirm the concept of cIMPACT-NOW Update 3 that patients diagnosed with IDH wild-type AA correlate prognostically with WHO grade IV tumors [25]. In the absence of an IDH mutation, the established histopathological gradation parameters necrosis and vascular proliferation indeed lose their prognostic significance even under similar therapeutic conditions of a combined radiochemotherapy. However, if IDH mutations can be detected in such tumors, the presence of necrosis and/or vascular proliferation still allows further prognostic differentiation according to the results of the study presented here.

\section{Acknowledgments}

We would like to thank the MTA Vanessa Dewor and Wiebke Schulze for their technical support. The project was founded by the Niedersächsische Krebsgesellschaft.

\section{Authors' contributions}

AC performed the molecular analysis, wrote parts of the manuscript. AA-H collected mainly the clinical data. RB performed the molecular analysis, wrote parts of the manuscript. UL \& SB performed parts of the molecular analysis. FB collected the clinical data, performed the statistical analysis. AB- $F$ collected the tissue. CS collected the tissue, wrote parts of the manuscript. VR collected the clinical data. FS generated the concept of the study, collected the clinical data. $\mathrm{CH}$ generated the concept of the study, performed the statistical analysis, wrote the manuscript. All authors read and approved the final manuscript.

\section{Competing interests}

The authors declare that they have no competing interests.

\section{Author details}

'Department of Neuropathology, Institute of Pathology, Hannover Medical School (MHH), Carl-Neuberg-Str. 1, D-30625 Hannover, Germany. ${ }^{2}$ Department of Neurosurgery, University Medical Center Göttingen, Göttingen, Germany. ${ }^{3}$ Department of Child and Adolescent Psychiatry, Charité Universitätsmedizin, Berlin, Germany. ${ }^{4}$ Institute of Pathology, Hannover Medical School, Hannover, Germany. ${ }^{5}$ Department of Neurosurgery, University Hospital Tübingen, Tübingen, Germany. ${ }^{6}$ Institute of Neuropathology, University Medical Center Göttingen, Göttingen, Germany. ${ }^{7}$ Department of Neuropathology, Institute of Pathology, University Medical Center Leipzig, Leipzig, Germany. ${ }^{8}$ Department of Neurosurgery, Städtisches Klinikum Dresden, Dresden, Germany.

Received: 7 August 2019 Accepted: 23 September 2019

Published online: 17 October 2019

\section{References}

1. Burger PC, Vogel FS, Green SB, Strike TA (1985) Glioblastoma multiforme and anaplastic astrocytoma. Pathologic criteria and prognostic implications. Cancer 56: 1106-1111 Doi. https://doi.org/10.1002/1097-0142(19850901)56: 5<1106::Aid-Cncr2820560525>3.0.Co;2-2

2. Barker FG, Davis RL, Chang SM, Prados MD (1996) Necrosis as a prognostic factor in glioblastoma multiforme. Cancer 77:1161-1166. https://doi.org/10. 1002/(sici)1097-0142(19960315)77:6<1161::aid-cncr24>3.0.co;2-z

3. Kleihues P, Burger PC, Scheithauer BW (1993) Histological typing of Tumours of the central nervous system. Springer Verlag, City

4. Kleihues P, Cavenee WK (2000) Pathology and genetics of tumours of the nervous system. IARC press; International Agency for Research on Cancer, City

5. Louis DN, Ohgaki H, Wiestler OD, Cavenee WK (2007) WHO classification of tumours of the central nervous system. International Agency for Research on Cancer, City

6. Louis DN, Ohgaki H, Wiestler OD, Cavenee WK, Ellision DW, Figarella-Branger D, Reifenberger G, von Deimling A (2016) WHO classification and grading of tumours of the central nervous system. IARC press; International Agency for Research on Cancer, City

7. Zülch KJ, Avtsyn AP, Barnar RO, Brucher JM, Earle KM, Fankhauser R, Ishida Y, Kunicki A, Olvera Rabiela JE, Rubinstein $\amalg$ et al (1979) Histological typing of Tumours of the central nervous system. Office of Publications, World Health Organization, Geneva, City

8. Wick W, Hartmann C, Engel C, Stoffels M, Felsberg J, Stockhammer F, Sabel MC, Koeppen S, Ketter R, Meyermann R et al (2009) NOA-04 randomized phase III trial of sequential radiochemotherapy of anaplastic glioma with procarbazine, lomustine, and vincristine or temozolomide. J Clin Oncol 27: 5874-5880. https://doi.org/10.1200/JCO.2009.23.6497

9. Weller M, Felsberg J, Hartmann C, Berger H, Steinbach JP, Schramm J, Westphal M, Schackert G, Simon M, Tonn JC et al (2009) Molecular predictors of progression-free and overall survival in patients with newly diagnosed glioblastoma: a prospective translational study of the German Glioma network. J Clin Oncol 27:5743-5750. https://doi.org/10.1200/JCO. 2009.23.0805

10. Hartmann C, Hentschel B, Wick W, Capper D, Felsberg J, Simon M, Westphal M, Schackert G, Meyermann R, Pietsch T et al (2010) Patients with IDH1 wild type anaplastic astrocytomas exhibit worse prognosis than IDH1-mutated glioblastomas, and IDH1 mutation status accounts for the unfavorable prognostic effect of higher age: implications for classification of gliomas. Acta Neuropathol 120:707-718. https://doi.org/10.1007/s00401-010-0781-z 
11. Brennan CW, Verhaak RG, McKenna A, Campos B, Noushmehr H, Salama SR, Zheng S, Chakravarty D, Sanborn JZ, Berman SH et al (2013) The somatic genomic landscape of glioblastoma. Cell 155:462-477. https://doi.org/10. 1016/j.cell.2013.09.034

12. Cancer Genome Atlas Research N, Brat DJ, Verhaak RG, Aldape KD, Yung WK, Salama SR, Cooper LA, Rheinbay E, Miller CR, Vitucci M et al (2015) Comprehensive, integrative genomic analysis of diffuse lower-grade Gliomas. N Engl J Med 372:2481-2498. https://doi.org/10.1056/ NEJMoa1402121

13. Capper D, Jones DTW, Sill M, Hovestadt V, Schrimpf D, Sturm D, Koelsche C, Sahm F, Chavez L, Reuss DE et al (2018) DNA methylation-based classification of central nervous system tumours. Nature 555:469-474. https://doi.org/10.1038/nature26000

14. Suzuki H, Aoki K, Chiba K, Sato Y, Shiozawa Y, Shiraishi Y, Shimamura T, Niida A, Motomura K, Ohka F et al (2015) Mutational landscape and clonal architecture in grade II and III gliomas. Nat Genet 47:458-468. https://doi. org/10.1038/ng.3273

15. Hegi ME, Rajakannu P, Weller M (2012) Epidermal growth factor receptor: a re-emerging target in glioblastoma. Curr Opin Neurol 25:774-779. https:// doi.org/10.1097/WCO.0b013e328359b0bc

16. Killela PJ, Reitman ZJ, Jiao Y, Bettegowda C, Agrawal N, Diaz LA Jr, Friedman AH, Friedman H, Gallia GL, Giovanella BC et al (2013) TERT promoter mutations occur frequently in gliomas and a subset of tumors derived from cells with low rates of self-renewal. Proc Natl Acad Sci U S A 110:6021-6026. https://doi.org/10.1073/pnas.1303607110

17. Hegi ME, Diserens AC, Gorlia T, Hamou MF, de Tribolet N, Weller M, Kros JM, Hainfellner JA, Mason W, Mariani L et al (2005) MGMT gene silencing and benefit from temozolomide in glioblastoma. N Engl J Med 352:997-1003. https://doi.org/10.1056/NEJMoa043331

18. Wick W, Meisner C, Hentschel B, Platten M, Schilling A, Wiestler B, Sabel MC, Koeppen S, Ketter R, Weiler M et al (2013) Prognostic or predictive value of MGMT promoter methylation in gliomas depends on IDH1 mutation. Neurology 81:1515-1522. https://doi.org/10.1212/WNL.0b013e3182a95680

19. Xu W, Yang H, Liu Y, Yang Y, Wang $P$, Kim SH, Ito S, Yang C, Wang P, Xiao MT et al (2011) Oncometabolite 2-hydroxyglutarate is a competitive inhibitor of alpha-ketoglutarate-dependent dioxygenases. Cancer Cell 19:1730. https://doi.org/10.1016/j.ccr.2010.12.014

20. Noushmehr H, Weisenberger DJ, Diefes K, Phillips HS, Pujara K, Berman BP, Pan F, Pelloski CE, Sulman EP, Bhat KP et al (2010) Identification of a CpG island methylator phenotype that defines a distinct subgroup of glioma. Cancer Cell 17:510-522. https://doi.org/10.1016/j.ccr.2010.03.017

21. Weller M, van den Bent M, Tonn JC, Stupp R, Preusser M, Cohen-JonathanMoyal E, Henriksson R, Le Rhun E, Balana C, Chinot O et al (2017) European Association for Neuro-Oncology (EANO) guideline on the diagnosis and treatment of adult astrocytic and oligodendroglial gliomas. Lancet Oncol 18: e315-e329. https://doi.org/10.1016/S1470-2045(17)30194-8

22. Stupp R, Mason WP, van den Bent MJ, Weller M, Fisher B, Taphoorn MJ, Belanger K, Brandes AA, Marosi C, Bogdahn U et al (2005) Radiotherapy plus concomitant and adjuvant temozolomide for glioblastoma. N Engl J Med 352:987-996. https://doi.org/10.1056/NEJMoa043330

23. Banan R, Christians A, Bartels S, Lehmann U, Hartmann C (2017) Absence of MGMT promoter methylation in diffuse midline glioma, H3 K27M-mutant. Acta Neuropathol Commun 5:98. https://doi.org/10.1186/s40478-017-0500-2

24. Lassman AB, Roberts-Rapp L, Sokolova I, Song M, Pestova E, Kular R, Mullen C, Zha Z, Lu X, Gomez E et al (2019) Comparison of biomarker assays for EGFR: implications for precision medicine in patients with Glioblastoma. Clin Cancer Res 25:3259-3265. https://doi.org/10.1158/1078-0432.CCR-18-3034

25. Brat DJ, Aldape K, Colman H, Holland EC, Louis DN, Jenkins RB, Kleinschmidt-DeMasters BK, Perry A, Reifenberger G, Stupp R et al (2018) CIMPACT-NOW update 3: recommended diagnostic criteria for "diffuse astrocytic glioma, IDH-wildtype, with molecular features of glioblastoma, WHO grade IV". Acta Neuropathol 136:805-810. https://doi.org/10.1007/ s00401-018-1913-0

26. Eckel-Passow JE, Lachance DH, Molinaro AM, Walsh KM, Decker PA, Sicotte H, Pekmezci M, Rice T, Kosel ML, Smirnov IV et al (2015) Glioma groups based on 1p/19q, IDH, and TERT promoter mutations in tumors. N Engl J Med 372:2499-2508. https://doi.org/10.1056/NEJMoa1407279

27. Sanson M, Marie Y, Paris S, Idbaih A, Laffaire J, Ducray F, El Hallani S, Boisselier B, Mokhtari K, Hoang-Xuan K et al (2009) Isocitrate dehydrogenase 1 codon 132 mutation is an important prognostic biomarker in gliomas. J Clin Oncol 27:4150-4154. https://doi.org/10.1200/JCO.2009.21.9832
28. Wijnenga MMJ, Dubbink HJ, French PJ, Synhaeve NE, Dinjens WNM, Atmodimedjo PN, Kros JM, Dirven CMF, Vincent A, van den Bent MJ (2017) Molecular and clinical heterogeneity of adult diffuse low-grade IDH wildtype gliomas: assessment of TERT promoter mutation and chromosome 7 and 10 copy number status allows superior prognostic stratification. Acta Neuropathol 134:957-959. https://doi.org/10.1007/s00401-017-1781-z

29. Ostrom QT, Gittleman H, Truitt G, Boscia A, Kruchko C, Barnholtz-Sloan JS (2018) CBTRUS statistical report: primary brain and other central nervous system tumors diagnosed in the United States in 2011-2015. Neuro Oncol 20:iv1-iv86. https://doi.org/10.1093/neuonc/noy131

30. Riemenschneider MJ, Reifenberger G (2009) Astrocytic tumors. Recent Results Cancer Res 171:3-24. https://doi.org/10.1007/978-3-540-31206-2_1

31. Shirahata M, Ono T, Stichel D, Schrimpf D, Reuss DE, Sahm F, Koelsche C, Wefers A, Reinhardt A, Huang K et al (2018) Novel, improved grading system(s) for IDH-mutant astrocytic gliomas. Acta Neuropathol 136:153-166. https://doi.org/10.1007/s00401-018-1849-4

32. Combs SE, Rieken S, Wick W, Abdollahi A, von Deimling A, Debus J, Hartmann C (2011) Prognostic significance of IDH-1 and MGMT in patients with glioblastoma: one step forward, and one step back? Radiat Oncol 6: 115. https://doi.org/10.1186/1748-717X-6-115

33. Costa BM, Caeiro C, Guimaraes I, Martinho O, Jaraquemada T, Augusto I, Castro L, Osorio L, Linhares P, Honavar M et al (2010) Prognostic value of MGMT promoter methylation in glioblastoma patients treated with temozolomide-based chemoradiation: a Portuguese multicentre study. Oncol Rep 23:1655-1662. https://doi.org/10.3892/or_00000808

34. Park CK, Park SH, Lee SH, Kim CY, Kim DW, Paek SH, Kim DG, Heo DS, Kim IH, Jung HW (2009) Methylation status of the MGMT gene promoter fails to predict the clinical outcome of glioblastoma patients treated with ACNU plus cisplatin. Neuropathology 29:443-449. https://doi.org/10.1111/j.14401789.2008.00998.x

35. van den Bent MJ, Baumert B, Erridge SC, Vogelbaum MA, Nowak AK, Sanson M, Brandes AA, Clement PM, Baurain JF, Mason WP et al (2017) Interim results from the CATNON trial (EORTC study 26053-22054) of treatment with concurrent and adjuvant temozolomide for $1 \mathrm{p} / 19 \mathrm{q}$ non-co-deleted anaplastic glioma: a phase 3, randomised, open-label intergroup study. Lancet 390:1645-1653. https://doi.org/10.1016/S0140-6736(17)31442-3

\section{Publisher's Note}

Springer Nature remains neutral with regard to jurisdictional claims in published maps and institutional affiliations.

Ready to submit your research? Choose BMC and benefit from

- fast, convenient online submission

- thorough peer review by experienced researchers in your field

- rapid publication on acceptance

- support for research data, including large and complex data types

- gold Open Access which fosters wider collaboration and increased citations

- maximum visibility for your research: over $100 \mathrm{M}$ website views per year

At BMC, research is always in progress.

Learn more biomedcentral.com/submissions 